\title{
TRABAJO NO CLÁSICO Y FLEXIBILIDAD
}

\author{
Enrique de la Garza Toledo*
}

\begin{abstract}
En este ensayo presentaremos el concepto de trabajo no clásico (De la Garza, 2010), como un intento de dar cuenta no solo de las diferencias en los tipos de trabajo, sino de dimensiones amplias de lo laboral incluidas en forma parcial en otras teorizaciones (Thompson, 1983). Buscaremos precisar el concepto y su alcance, así como las relaciones con las actividades de servicios, que se han vuelto cada vez más importantes en las economías modernas, sin olvidar su peso en las menos desarrolladas. Entraremos a recapitular sobre el concepto clásico de flexibilidad (Durand, 2004) y la ampliación que sería pertinente al incluir trabajos no clásicos. En particular incluiremos una forma de flexibilización que probablemente se esté convirtiendo en dominante en el capitalismo luego de la última gran crisis económica, la subcontratación (Moncada y Monsalvo, 2000) y sus vínculos con el trabajo no clásico. Finalmente, discutiremos las tesis de la fragmentación de identidades (Sennet y Coob, 1972) y la servidumbre voluntaria (Durand, 2006) al calor del enfoque mencionado de trabajo no cásico, para culminar con consideraciones acerca de la posibilidad de constitución de sujetos laborales en estas condiciones. Palabras Clave: Trabajo. Trabajo clásico. Trabajo no clásico.
\end{abstract}

\section{INTRODUCCIÓN}

El concepto clásico de trabajo, en el sentido de la forma teórica que adoptó en muchas ciencias sociales este tipo de actividad, se vincula con la Revolución Industrial (Thompson, 1972), cuando el capitalismo adquirió una forma moderna y cuando en algunos procesos productivos centrales fueron introducidas las máquinas para la realización de la transformación de la materia prima, impulsadas no por la fuerza de los obreros sino, inicialmente, por la del vapor. Es lo que Marx llamó la fase maquinista de la transformación de los procesos productivos capitalistas. Estos procesos productivos implicaban también el uso de trabajo asalariado por el capital, la subsunción real del primero no solo al capital sino al ritmo de las máquinas; también la segmentación espacial (la fábrica) y temporal (jornada de trabajo) entre el

* Doutor em Sociologia. Professor da Universidade Autônoma Metropolitana, Departamento de Sociologia. Professor visitante da Universidade Autônoma de Barcelona, Universidade de Cornell e de Evry (França).

Caixa Postal 55536, 09340, México, D.F., Purísima y Michoacán, Col. Vicentina, Edificio H-141 egt57@hotmail.com mundo del trabajo y otros mundos de vida de los trabajadores (Moore, 1995). Este tipo de trabajo sirvió de basamento empírico a teorías muy diversas en cuanto a los conceptos de lo que es trabajo, producción, producto, relación laboral. Por ejemplo, para la teoría neoclásica solo sería trabajo el que produce para el mercado, con esto se incluye al trabajo no asalariado que produce para el mercado, pero el eje de la teorización sería la producción capitalista fabril. Otro tanto sucede en el marxismo, con el añadido de que trabajo no es solo transformación de objetos de trabajo a través del trabajo para el mercado sino que también puede incluirse el que produce para el autoconsumo. Sin embargo, casi toda la teorización de El Capital sin duda que tiene como referente a la producción capitalista maquinizada. En su forma más desarrollada, en torno del capital los conceptos de valor, valor de uso, mercancía y dinero, excepto observaciones al margen, los conceptos de esta obra se refieren a dicha producción (capital constante, variable, plusvalía, acumulación de capital, rotación, etc.). Implicaba también una separación temporal y espacial entre producción (fábrica), 
circulación con puntos de venta fuera de la fábrica y consumo en otras empresas o en los hogares (De la Garza, 2011). Es decir, el cliente aparecía en el mercado y en el consumo pero no directamente en el proceso productivo. Este implicaba un acercamiento que solo permitía la relación directa entre dos sujetos y sus representantes, el del trabajo y el del capital.

Otro tanto podríamos decir de las teorías originarias de la Sociología del Trabajo (Mayo, Friedman, Touraine, Naville, Golthorpe, Panzieri, Braverman) centradas en el proceso de producción capitalista de tipo industrial; o bien las teorías de organizaciones o la psicología del trabajo (De la Garza, 2006).

La apuesta a ver a la producción industrial capitalista, inicialmente maquinizada, como el paradigma de toda producción no era gratuito. Mostraba que efectivamente, hasta los años sesenta del siglo XX, decir capitalismo era casi sinónimo de industrialización. En esta medida fueron creados los conceptos centrales de las teorías que analizan el trabajo teniendo como referencia al sector industrial y sus transformaciones. Hubo diversas formas de conceptualizar las transformaciones del trabajo capitalista al industrial, pero una de las más simplificadas era la que distinguía etapas históricas en el desarrollo de los modelos de producción capitalistas. Comenzando por el trabajo de oficio capitalista - no hay que confundirlo con el artesanal puesto que se realizaría con trabajadores asalariados del capital - en procesos que Marx llamó "de cooperación simple y manufactureros” (Offe, 1998). No se entendía por tales, como ahora la economía hegemónica, un sector de la economía, sino procesos capitalistas no maquinizados dependientes de las habilidades de los trabajadores. Luego vendría el trabajo subordinado a las máquinas y, posteriormente, con la introducción primero del Taylorismo y luego del Fordismo, se completaría la subsunción real del obrero al capital, pero no solo a la máquina sino también a la organización del trabajo (obrero masa, descalificado, que realiza trabajo segmentado, rutinario, monótono). La Gran Crisis de los
Setenta del siglo XX habría conducido a una profunda reestructuración de las grandes empresas, primero en el sentido del Toyotismo (reintegración de tareas a través de la polivalencia, el trabajo en equipo, la calidad total, el control estadístico del proceso y el justo a tiempo, trabajadores recalificados, involucrados, con iniciativa en la toma de decisiones en el puesto de trabajo) (Burawoy, 1979), junto con la introducción de la computación en el control del proceso productivo y de toda una planta o conjunto de plantas; primero en las tareas productivas, luego también en diseño, compras, ventas, contabilidad, finanzas. Todo esto sin olvidar la importancia que algunos autores dan en este proceso a la formación de clusters y otras redes entre empresas.

Con diferentes conceptos y salvedades acerca de cómo definir las etapas, esta sería más o menos la línea de desarrollo clásico de la producción industrial, a veces extendida a servicios modernos e incluso a una parte de la agricultura.

Este trabajo clásico siempre convivió con otras formas laborales (artesanal, de oficio, en servicios, agricultura no industrial), pero se pensó que en el PIB la parte más importante iría correspondiendo a la industria e incluso en el empleo. Y así fue hasta 1950. Sin embargo, a partir de este año en los Estados Unidos y otros países europeos los servicios comenzaron a crecer y actualmente en la mayoría de estos representan la mayor parte del PIB y del empleo. En países no desarrollados la importancia de los servicios no es nueva, pero una parte muy elevada de estos corresponde a los servicios precarios, incluso informales que existían de larga data. Es decir, lo clásico o lo no clásico no se relaciona con su importancia en el empleo o en el producto, tampoco con su antigüedad histórica (Handy, 1986).

Muchos conceptos se han propuesto como alternativos a lo que llamamos trabajo no clásico (García, 2006). Uno de los primeros fue el de trabajo informal (Portes, 1995; Tokman, 1987), que en su acepción original (misión de la OIT a Africa en los setenta del siglo pasado) apuntaba a un concepto colindante con el más actual de modelo de 
producción porque incluía tecnología, organización, relaciones laborales, características de la mano de obra, relación con la unidad doméstica. Esta línea, desde nuestro punto de vista, hubiera sido más fructífera que las actuales, enfrascadas en cómo medir la informalidad, que las ha llevado a simplificar lo que empezó como un concepto muy complejo. Las dos definiciones más socorridas actualmente son: sector informal, que no lleva una contabilidad racional separando las cuentas de las familia de las del negocio, que no está registrada y que no paga impuestos; y la de relación laboral informal, relativa a los trabajadores con los que no se cumplen las protecciones de las leyes laborales, específicamente derecho a la salud por ser trabajador y a la pensión. Es decir, estas definiciones están centradas en la relación de la unidad económica o del trabajador, con instituciones externas al trabajo (fisco y seguro social), pero no en el contenido del trabajo que es lo que nos interesa destacar, como veremos con el concepto de trabajo no clásico (Salas, 2006).

Otro tanto sucede con los conceptos de trabajo no estructurado, concepto poco acertado puesto que las estructuraciones del trabajo no pueden quedar reducidas al cumplimiento de regulaciones legales. Trabajo atípico (Senise, 2001), nuevamente se centra en el cumplimiento o no de las regulaciones laborales. Precario, muy centrado en variables sociolaborales (inestabilidad, inseguridad o falta de protección social, vulnerabilidad social y económica) más que en el contenido del trabajo, trabajo no estándar (Reglia, 2003), que mira hacia protecciones legales o no. Trabajo decente, con el que se amplía la mirada de las protecciones hacia el derecho a sindicalizarse, a firmar contratos colectivos, seguridad social, diálogo social. Exclusión, vulnerabilidad e inseguridad, apuntan no tanto a derechos no cumplidos sino a la relación del trabajador con la sociedad, desarticulación de relaciones sociales entre individuos, pérdida de solidaridad, fragilidad del vínculo social; el trabajo deja de articular el tiempo cotidiano. Estos últimos conceptos colindan con los que criticaremos más delante de fragmentación de las identidades.
Es decir, ninguno de estos conceptos, acuñados en los últimos tiempos - el de informalidad es el más antiguo - nos ayuda a dar cuenta del cambio en los contenidos del trabajo, del producto, de las relaciones sociales en el trabajo, independientemente de que se cumpla lo dispuesto en las leyes laborales.

El concepto más antiguo que sirve de inspiración al de trabajo no clásico es el de Marx asociado a la producción inmaterial. En la Historia Crítica de las Teorías sobre la Plusvalía Marx acuña con gran perspicacia este concepto, notando desde su época que hay trabajos que no se ajustan exactamente a su propia teorización sobre el trabajo industrial. Un ejemplo que utiliza es la representación de una obra de teatro en donde el teatro es propiedad de un capitalista, los actores son asalariados y el público paga por el espectáculo y el negocio debe generar ganancias para sostenerse y acumular capital. Según Marx, en este tipo de producción, primero el producto es inmaterial y no material como lo es en la industria. Marx como buen filósofo no entendía por material solo lo físico material, que está diferenciado del productor y puede ser observado a través de los sentidos, sino entiende por material lo objetivado; es decir que, aunque es producto del trabajo humano adquiere una existencia separada de su productor. Sin embargo, en la obra de teatro en un solo acto se produce el espectáculo (que no es sino una configuración de símbolos que adquieren significados para los espectadores), al mismo tiempo se circula como mercancía hacia los compradores que son los espectadores y se consume en el mismo teatro por estos. Es decir, la producción simbólica que es la obra termina subjetivándose en el espectador y no puede ni almacenarse ni revenderse. El producto no se objetiva sino se subjetiva. Para nosotros este sería un primer tipo de trabajo y producción no clásica, pero que no lo agota. También puede haber una producción puramente simbólica objetivada, es decir, los símbolos generados adquieren una existencia separada de su productor (el diseño de software, el film, el libro, etc.). De acuerdo con el concepto de 
materialidad expuesto, esta producción no sería inmaterial sino material pero eminentemente simbólica - por supuesto que a toda producción eminentemente simbólica siempre se le asocia algo físico material, la depreciación del teatro, el CD, el costo del material del libro, pero esta parte de su valor no es lo que lleva a ser comprado sino su contenido simbólico.

Dentro del trabajo no clásico también estamos incluyendo el trabajo eminentemente interactivo en el sentido no de que se interacciona para trabajar (esto es propiedad de todos los trabajos sean cara a cara o virtuales), sino que la interacción es la condición para producir y, al mismo tiempo, lo más importante del producto. Trabajo interactivo lo llaman algunos, válido siempre y que se acote que todos lo son, pero no en todos es parte de lo que se vende (cuidado de bebés, de ancianos, educación tradicional, cuidado en hospitales, parte importante en las ventas directas al cliente, en el trabajo de call centers, en comida rápida, el del taxista, etc.). Si en cierto tipo de trabajos es muy importante la forma de interacción con el cliente, esta triada puede ampliarse a más sujetos, especialmente cuando se trabaja en el espacio público (taxistas que interaccionan con el pasajero, con agentes de tránsito, automovilistas, peatones). Estas interacciones pueden ser cara a cara o virtuales a través de teléfono, internet. Habría que añadir que toda interacción entre sujetos es simbólica pues implica la generación e intercambio de símbolos que son transformados en significados en la subjetividad de los involucrados. En otras palabras, el trabajo interactivo es también trabajo de generación y transmisión de símbolos y de allí las dificultades del consenso en lo que se quiso decir. Es decir, una parte del trabajo es creación de símbolos comprensibles para el otro, que impacta nuevas calificaciones de los trabajadores para lograrlo e implica a un externo a la relación capital trabajo, clásica en esta actividad. En esta medida, en el trabajo no clásico, por su énfasis en lo simbólico - no reducido a lo cognitivo sino que implica además lo emocional, moral, estético-, con mayor razón cuando es interactivo, el concepto de trabajo - que es trabajar, como se trabaja y que se produce - tiene que implicar cómo se generan los símbolos, y cómo interviene el cliente u otros sujetos como en el caso del taxista en esa construcción simbólica. La Sociología del Trabajo, al menos desde inicios de los ochenta, acepta el concepto de trabajo emocional; de fines de los noventa el de trabajo estético y con mayor facilidad aceptó el de trabajo cognitivo relacionado con la ciencia y la tecnología y la innovación (Micheli, 2006). Si bien estas denominaciones indican énfasis en el tipo de códigos o símbolos producidos, nunca pueden presentarse solos, lo que habla de la necesidad no de tipologías de trabajo no físico material sino de cómo se combinan en diferentes configuraciones y con énfasis diversos los códigos cognitivos, emocionales, estéticos, morales y se conforman en red (configuración) (De la Garza, 2001) a través de formas de razonamiento lógico formal, pero también del razonamiento cotidiano (metáfora, analogía, regla práctica, hipergeneralización, retórica, etc.). Es decir, cómo, para la actividad concreta de trabajar, se construyen configuraciones concretas de códigos o símbolos que no dependen solo del trabajador sino también de la actividad y consenso del cliente o de otros actores, según el caso. Es decir, el cliente en estos trabajos no clásicos no puede verse como un ente pasivo que compra y consume, porque lo que compra no se llega a generar sin su propia actividad. A pesar de no ser asalariado de la empresa que le vende, en esta medida, dentro de la idea de extensión de conceptos habría que considerar en este tipo de trabajos el del cliente (Jurgens, 1995). Trabajo no asalariado que si no se realiza no se tiene el producto: la compra en supermercados, en un restaurante de hamburguesas. Por esta razón, en los diseños organizacionales de cadenas de supermercados, cines, comida rápida, se contempla la actividad del cliente como parte de lo que permitirá la generación del servicio.

Una última dimensión del trabajo no clásico (simbólico, interactivo) es el tema de los traslapes entre espacio convencional del trabajo y otros 
mundos de vida, sea del propio trabajador, de los clientes o de otros actores que intervienen, sin ser trabajadores ni compradores, en el proceso de trabajo (De la Garza, 1997). Es el caso del trabajo a domicilio, sea tradicional (costurera) o moderno (teletrabajo en casa). Trabajar es también poner en juego la imbricación de espacios, de tal forma que el espacio y el tiempo de trabajo no se pueden separar de esas imbricaciones (para el taxista detenerse a comer durante la jornada en un restaurante es parte de su jornada y de las actividades laborales) (Pogliaghi, 2011).

El concepto no clásico de trabajo debe ser considerado como un concepto ampliado, tanto en el nivel de la valorización como en nivel del proceso de trabajo. Desde este último nivel habría que pensar en ampliar, como hemos mencionado, el concepto de que es trabajar y quien trabaja, pero también el de control sobre el trabajo. Al estar en interacción con no asalariados (el cliente, pero también pueden ser otros actores dependiendo de cual trabajo se trate) estos también ejercen control sobre el trabajo del asalariado, además del patrón. En esta medida se impacta también el concepto de relación laboral - entendida estrictamente como relación social en la producción - que en el clásico queda reducida, cuando se trata de trabajo asalariado, a la del capital con el trabajo, pero en el no clásico esta relación puede ser tríadica (inclusión del cliente) o poliádica (interviene otros agentes favoreciendo u obstaculizando el trabajo) (Muckenberger, 1996). Y se impacta también el concepto de construcción social de la ocupación, que no depende solo de quien quiere trabajo y quien necesita trabajadores (oferta y demanda de trabajo) sino que pueden intervenir otros actores, redes sociales, además de la propia subjetividad de los que intervienen (Zucchetti, 2003). Finalmente la imbricación de espacios de relaciones puestas en juego, al mismo tiempo que se trabaja, puede requerir conceptos bisagra que den cuenta de esos espacios, a la vez que ya no aparecen segmentados ni espacial ni temporalmente.

En síntesis entendemos por trabajo no clásico aquel en el que la intervención del cliente es indispensable para que se realice la producción y se tenga el producto. Ya sea porque se generan símbolos y se transmiten al cliente, o porque el producto es la interacción misma. La intervención del cliente implica interacción con los trabajadores clásicos y, a veces, con otros actores aparentemente ajenos a dicho trabajo, e intercambios simbólicos entre los sujetos del trabajo, incluyendo al cliente. Esto porque parte importante del trabajo no clásico es la producción e intercambio de símbolos (cognitivos, emocionales, morales, estéticos).

En última instancia el concepto de trabajo no clásico puede ser más que un tipo de trabajo, un enfoque de análisis.

\section{LOS SERVICIOS}

En el centro de los trabajos no clásicos están los servicios, aunque puede incluir trabajos en la industria y la agricultura. Su definición sigue siendo objeto de controversia. Sin embargo, las dificultades de la definición dependen también del enfoque de análisis del fenómeno laboral que se adopte. En el enfoque sociodemográfico y económico, en el que se trata de relacionar variables propias de cualquier tipo de trabajo (edad, escolaridad, nivel educativo, estado civil, duración de la jornada laboral, antigüedad en el trabajo, salario, disposición de prestaciones, etc. ) no importaría si la producción fuera material o inmaterial, física o simbólica, interactiva con creación de significados, pues las variables solo se diferenciarían en nivel entre los diversos trabajos, de tal manera que la diferenciación entre trabajo clásico y no clásico sería ociosa. Así mismo, para la perspectiva jurídica lo fundamental es la observancia de la norma, independientemente del tipo de trabajo, salvo excepciones contempladas en la misma Ley. De tal manera que las diferencias mencionadas en el Apartado I serían irrelevantes. En cambio en las tradiciones de la Sociología del Trabajo, la Antropología, la Psicología, Las Relaciones Industriales, la Administración y las Organizaciones es, o puede ser, muy importante 
analizar el trabajo como actividad por la cual se transforma una materia prima, a través de la fuerza de trabajo, utilizando ciertos medios de producción (Korezynski, Hodson y Edwards, 2006). Es la perspectiva de analizar el trabajo en acción en el proceso de trabajo, la cual puede tener importancia para entender el comportamiento productivo de las empresas, así como para aquellos que piensan que el trabajo no es un simple factor de producción o costo laboral, sino sujetos en acción dotados de subjetividad que interaccionan en los procesos de trabajo y generan productos. En esta medida, cuanto trabajo incorporado al producto generará la fuerza de trabajo, no está determinado por su salario. El trabajador puede generar más valor de lo que su fuerza de trabajo cuesta. De tal manera que su productividad se defina en las relaciones cotidianas de producción, día por día, dependiendo de la resistencia o cooperación del trabajador con la gerencia. Pero, la perspectiva de análisis del trabajo en el proceso de trabajo puede también orientarse en cuanto a la posible formación de sujetos colectivos de trabajadores, como veremos en la última parte de este ensayo.

En esta tesitura, los servicios han sido primero definidos como un residuo, lo que queda en el producto nacional luego de descontar a la industria, agricultura, pesca, silvicultura, ganadería. Los que han tratado de darle contenido analítico a la definición de servicios han comenzado por considerarlo como el sector de producción de intangibles. El concepto de intangible a estas alturas resulta muy elemental para dar cuenta de la complejidad de los servicios. Primero, porque remite a uno sólo de los sentidos del cuerpo, el del tacto, lo que se puede o no tocar, por ejemplo, la música se oye pero no se toca. Sin embargo, la comida en el restaurante si se puede tocar y se considera servicio, aunque el buen trato del personal "no se puede tocar". El ejemplo lo que indica es que los servicios comúnmente combinan producto material con inmaterial, aunque con un peso importante de lo inmaterial, de lo simbólico y/o de lo interactivo. Otros han tratado de reducir los servicios a los que están basados en el conocimiento. Nuevamente, no podríamos encontrar aislado conocimiento de emoción, moral y estética, por decir algo, aunque uno de estos campos simbólicos podría tener más peso. En todo caso, en el placer por la música lo más importante no es el conocimiento sino la emoción y lo estético. También hay quienes han definido los servicios como interactivos directamente, y hay muchos servicios así (restaurante, concierto en vivo). Pero, hay servicios sin interacción directa entre trabajadores y clientes, como en buena parte de las telecomunicaciones. Finalmente, se les ha definido como productos no almacenables, aunque los paquetes de cómputo si lo son.

En otras palabras: puede haber intangibles objetivados (software); servicios que ofrecen un tangible (comida en restaurante); tangibles con fases intangibles en su producción (diseño en automotriz); intangibles observables (concierto); la percepción de tangibles es, a su vez, un intangible; una parte de lo material es intangible. Resultan más pertinentes, ante estas confusiones, las diferencias entre material einmaterial, observable einobservable, interactivo cara a cara y mediatamente.

Es decir, resulta más conveniente una definición bidimensional de lo que es un servicio: producción eminente de símbolos y/o producción de interacciones con los clientes y otros actores con significado, es decir, el centro del trabajo no clásico estaría en los servicios definidos de esta manera. Aunque una parte de la producción material puede ser no clásica al crecer la importancia del cliente y de las interacciones como en la preparación de hamburguesas en un McDonald's.

\section{FLEXIBILIDAD Y TRABAJO NO CLÁSICO}

El tema de flexibilidad del trabajo se ha vuelto un lugar común a partir de la última década del siglo pasado para analizar las relaciones laborales. La gran crisis capitalista de mediados de los setenta de dicho siglo fue atribuida por sectores pro empresariales a la rigidez de las relaciones laborales, resultado del largo período de 
Estado benefactor y keynesiano, que implicó pactos entre Estado, sindicatos y empresas. En esta medida había que flexibilizar el mercado laboral. Aunque el término de flexibilización no era usado en la economía neoclásica, resultaba fácil asimilarlo al concepto de eliminar rigideces al libre encuentro entre oferta y demanda de trabajo. Es decir, el centro estaba en el mercado de trabajo. Sin embargo, las primeras reestructuraciones productivas no fueron tanto al mercado sino al proceso de trabajo, rescatando la tradición de las grandes empresas automotrices japonesas y que luego se llamaría el Toyotismo. En otras palabras, aunque parecieran referirse a lo mismo los que hablan de flexibilizar el mercado y el proceso de trabajo, las connotaciones son diferentes. Entre estas dos posiciones empresariales se encontraba la idea de flexibilizar el sistema de relaciones industriales. La primera y la tercera fueron asimiladas por las propuestas neoliberales al coincidir con la economía neoclásica, siendo traducidas como eliminar rigideces (protecciones) contenidas en las leyes laborales y en la contratación colectiva, así como establecer limitaciones a los pactos con los sindicatos, en especial marginarlos del diseño de políticas económicas, laborales o sociales. En otras palabras, se trataba de flexibilizar un sistema de relaciones industriales relativamente benefactor del trabajo y, de esta manera, permitir que salarios y empleo se fijasen por productividad marginal del trabajo y oferta y demanda del mismo. Aunque relacionada con la flexibilidad en el proceso de trabajo, desde el momento en que este obedecía a normas de las relaciones laborales internas y externas de la ley laboral, se trataba de facilitar el empleo y el despido (flexibilidad numérica), la movilidad interna de los trabajadores entre puestos, categorías, departamentos (flexibilidad funcional) y poner el salario en función de la productividad (la calidad, la puntualidad y asistencia, el tener menos desperdicios), a las que habría que añadir la flexibilidad a través del outsourcing (Uriarte y Tusso, 2009; Novella et al., 2007; Martínez, 2008).

Todo esto fue pensando directamente para el sector industrial, aunque extendido a los servicios. En la doctrina estrictamente toyotista, como era importante la identidad con la empresa, el trabajo en equipo, el involucramiento y la participación de los trabajadores para aumentar la productividad, no necesariamente la máxima flexibilidad daría la máxima productividad; la nueva cultura laboral se consideraba muy importante, en particular cuando entraba a jugar, además de la productividad, la calidad. Estas concepciones, especialmente las neoclásicas, de entender la flexibilidad como desregulación, han estado presentes en el centro de las disputas entre el capital y el trabajo en el nivel internacional desde los años ochenta hasta la actualidad.

El problema es que los conceptos de desregulación y de flexibilidad neoclásica y toyotista fueron creados mirando al sector industrial (Piore y Sabel, 1988). El problema es cómo se presenta para el trabajo no clásico que implica producción eminente de símbolos con intervención inmediata o mediata del cliente (además de otros posibles actores) y la interacción entre estos. En el caso de la producción físico material clásica la disputa directa puede ser capital-trabajo en torno a obligar o convencer al trabajador de ser más productivo (valor agregado/hora-hombre). En este caso el trabajador se puede resistir o cooperar. En la producción no clásica el problema es que el cliente que intervine no puede ser controlado estrictamente por la gerencia, como se intenta con el trabajador por estar este al mando del capital. El cliente puede cooperar o no, pero también puede imponer durante la interacción productiva variaciones no estandarizadas que rompen la rutina y pueden afectar la productividad y la calidad. En esta medida una extrema rigidez en los ordenamientos de cómo trabajar puede ser contraproducente para la productividad y sobre todo para la calidad. Asimismo, la producción de símbolos compartidos entre trabajador y cliente pueden entrar en desacuerdo más allá de que el trabajador se comporte de acuerdo con la normatividad empresarial (Hochschild, 1983). Además, se presenta el problema de qué tanto puede estandarizarse la 
producción y el intercambio de símbolos, en parte porque el cliente no es estrictamente controlado, pero también porque no hay cadenas de montaje de símbolos para formar configuraciones, ni mucho menos tiempos estándar para genera un símbolo compartido. En el caso de la producción objetivada de símbolos (producción de software), sin intervención directa del cliente (aunque puede intervenir directamente en el llamado software a la medida), el problema es nuevamente si se puede estandarizar las operaciones cognitivas y fijar tiempo estándar de producción. Ilustraremos este caso con la producción de software:

El diseño de software es un ejemplo de producto simbólico objetivado: el programa existe al margen de su creador y sus consumidores pueden intervenir directamente en el proceso de trabajo (software a la medida) o bien a través de la demanda; se puede almacenar y revender; el proceso de trabajo usa como insumos símbolos o vocablos del lenguaje de programación y algoritmos; las operaciones más importantes de transformación se dan en la subjetividad del programador y son de carácter cognitivo; se producen en un campo de interacciones entre gerencia, líder del proyecto y programadores. Lo que implica consensos acerca de cuál camino seguir, aunque es el programador el que decide la secuencia de códigos a utilizar para resolver el problema. Es decir, en la solución del problema influye la habilidad del programador, la cual es de resolución simbólica de problemas, aunque supone colaboración con líderes y gerencia, también con probadores de la calidad e incluso con comunidades virtuales de programadores que se ayudan más allá de las fronteras de la empresa. Las metodologías que sigue el programador no son lineales, no aceptan en general su asimilación a reglas burocráticas y siempre hay incertidumbre en cuanto a si se tendrá la solución, si habrá errores y sobre todo respecto al tiempo de producción. En esta medida, para evitar la "aflicción de software" (errores en el mismo y falta de tiempos estándar) aparecen propuestas de estandarización y rutinización en el diseño como la Ingeniería del Software. Es una propuesta aná- loga a la de la administración científica del trabajo de Taylor para la manufactura, aunque en este caso el intento es estandarizar tiempos y pensamientos y no movimientos. Esta perspectiva se enfrenta a las limitaciones que provienen de la incertidumbre del proceso que no se reduce al ensamble de partes de cadenas de códigos, las soluciones están muy relacionadas con las habilidades "artesanales" de los programadores y las soluciones muchas veces no son generalizables sino son ad hoc. También se enfrenta la estandarización al hecho de que los programadores no documentan en forma suficientemente explícita la manera como diseñaron software previos, a veces por la lucha por el saber-pensar y el intento del monopolio del conocimiento por parte del diseñador. Por estas razones el diseño de software se ha resistido a su estandarización y al establecimiento estricto de tiempos estándar para su producción. Una solución paralela para lograr la estandarización en el diseño de software ha sido la separación entre lo más importante de la concepción del diseño a cargo de la gerencia, dejando las tareas menores de codificaciones de logaritmos a los programadores. Todos estos intentos solo han fructificado parcialmente y la incertidumbre sigue en esta actividad.

En este ejemplo se puede observar que la producción de símbolos tiene una parte de creatividad en la concepción que no puede reducirse a rutinas, sino que depende de las habilidades subjetivas de este tipo de trabajador, así como de las relaciones que se entablen con la jerarquía, de cooperación o conflicto con la empresa. Estas circunstancias marcan límites no solo a la estandarización sino al control de la empresa de los tiempos de producción e incluso de la calidad del producto. No es posible estandarizar radicalmente las redes neuronales-además de que, si existieran estas es imposible ubicarlas con precisión y mucho menos reconfigurarlas planeadamente para producir diseños en menos tiempo y de mejor calidad - que supuestamente se pondrían en funcionamiento para el diseño; ellas tienen que ver con la biología y la experiencia, con el conocimiento pero también con otros cam- 
pos de la subjetividad del diseñador, así como con estructuras y relaciones. En el caso de trabajo no clásico es difícil pensar en la taylorización de la asimilación de códigos y la creación de significados estrictamente a voluntad, así como el establecimiento de interacciones proactivas siempre, erradicando el conflicto y la competencia entre los propios trabajadores o con los mandos. No es posible separar tajantemente concepción y ejecución en el trabajo simbólico pues todo trabajo simbólico implica concepción y mucho menos reducirlo a microoperaciones simbólicas de transformación claramente identificable, todo lo cual dificulta la aplicación de estandarización, rutinización, simplificación de las tareas de simbolización y también la constitución de cadenas de montaje de símbolos, estrictamente sincronizadas. En estas actividades de creación de símbolos puede ser importante el trabajo en equipo, pero cada uno de sus miembros tendrá funciones menos claras que en la producción material; en estas relaciones juegan más las interacciones simbólicas para lograr la cooperación, de tal forma que el control total de la calidad, muchas veces no puede funcionar para operaciones parciales sino para resultados y el justo a tiempo puede ser un concepto exótico por lo que mencionamos. Es decir, en este tipo de producción no basta, aunque importa, el Toyotismo, para lograr productividad y calidad, y la tecnología es un instrumento que no resuelve los problemas principales. En cambio, la dependencia del producto $y$ del proceso respecto al trabajador y sus cualidades, potencia sus capacidades de resistencia. En el diseño de software los programadores pueden ocultar códigos, es decir no explicar con detalle cómo llegaron a la solución, lo que les da el monopolio sobre ese conocimiento y los hace menos sustituibles. De la misma manera, la formación de redes virtuales de solidaridad posee componentes personales que no son estrictamente transferibles a otros.

Cuando en la generación de software interviene el cliente, como en el llamado software a la medida, la situación se complica. El cliente se encuentra en interacción en varios momentos con el programador durante el diseño del software. En este caso, se amplían las interacciones, cooperaciones o conflictos con un tercero en discordia que no es empleado ni directivo. Estas relaciones influyen en las soluciones, en los tiempos y calidades, así como en el control del proceso de trabajo, complicando la relación social de trabajo a tres partes.

Otra situación de trabajo no clásico es el restaurante de hamburguesas (McDonald's). Este es un caso que combina un trabajo interactivo y significativo con otra parte de producción físico material. La segunda es propiamente la preparación de la hamburguesa que sigue un proceso taylorizado y maquinizado, con participación lateral del sistema informático para transmitir las órdenes. El trabajo de los empleados es poco calificado, rutinario (se producen pocos productos estandarizados, sencillos, en masa, para su venta al menudeo, se trabaja en condiciones precarias). Esta parte no se diferencia de la producción de ropa en maquiladoras. Sin embargo, la parte manufacturera está íntimamente imbricada con la del servicio de venta en el propio restaurante y el consumo en el mismo, puesto que el producto se genera por pedido del cliente y no se almacena. Para ser atendido el cliente tiene no solo que pagar, sino escoger, formarse, ordenar con precisión (siguiendo ciertas frases ya hechas por la empresa), esperar a que se surta su orden, tomar el producto, sentarse, consumir y depositar los desperdicios en recipientes para tal efecto. Es decir, aunque el cliente no es un empleado, ni tampoco un directivo tiene que "trabajar" para lograr la compra y el consumo. De tal forma que la empresa hace diseños organizacionales que incluyen al cliente que no es su empleado: mostrador y caja registradora especialmente diseñada para que el cliente haga una fila, pizarrones con la lista limitada de productos para que no se pierda el tiempo usando otro lenguaje que confunda al empleado o evitar las preguntas ambiguas como "¿Qué hay de comer?". Los asientos donde se consume están diseñados para no hacer agradable permanecer mucho tiempo y los contenedores de desperdicios 
se diseñan para que se depositen estos con facilidad e inmediatamente las charolas. Es decir, se hace un diseño del espacio, de los instrumentos de acarreo de las hamburguesas y para el depósito de desperdicios que ahorran tiempo de trabajo del cliente. Esto es porque si el cliente no trabaja no se realiza el servicio o si lo hace en forma torpe o perezosa ocupa espacio y tiempo que afecten la eficiencia del restaurante. En otras palabras, el trabajo en este tipo de negocios no puede desentenderse del cliente. Este trabaja en los pedidos, circulación y consumo que normalmente se realizan en el mismo espacio que se da la producción de la hamburguesa. En este proceso importan las interacciones entre el cliente y algunos de los empleados, al momento de ordenar y pagar; interacciones superficiales y estereotipadas que, sin embargo, pueden sufrir de muchas disrupciones imprevisibles y afectar con esto al proceso global de producción-circulación y consumo de la hamburguesa. Es decir, el empleado puede ser controlado en forma más o menos estricta por la gerencia en cuanto a la fase de producción físico material, a través de gerentes de restaurante, supervisores o el cliente misterioso, pero no puede controlar estrictamente la interacción del empleado con el cliente, porque no depende solo del primero, que puede estar muy bien capacitado para estandarizar dicha interacción, porque el cliente puede salirse del guión y en el peor de los casos la consecuencia puede ser que no sea atendido. Lo mismo sucede con cada operación que el cliente tendría que realizar con su trabajo para que el servicio se realice con eficiencia (el cliente puede no depositar los desperdicios en el recipiente adecuado). El empleado sufre la presión de la empresa a través de gerentes y supervisores, pero también del cliente que puede protestar si considera deficiente el servicio. Es decir, el cliente en parte controla al empleado y en parte es controlado por la empresa en el intento de taylorizar su trabajo.

En este caso, como en el del diseño del software, la relación de trabajo se complica con respecto del trabajo clásico, en esta relación interviene el cliente en varios momentos y se convierte en una tríadica entre asalariado, directivo y cliente. Otro tanto pasa, como decíamos con el control y quien controla al trabajador. El taylorismo es una realidad en la fabricación de la hamburguesa, se puede combinar con principios de toyotismo, pero la relación del cliente es de una persona con un trabajador, comúnmente el que recibe los pedidos y cobra. Aunque se presiona al cliente para que emplee poco tiempo en el consumo esto no puede garantizarse y no hay circulación y consumo justo a tiempo, ni tampoco se puede garantizar con el establecimiento de palabras y frases estándar la compresión mutua entre cliente y empleado. El empleado es en buena medida, en cuanto a la transformación físico material de la carne en hamburguesa un obrero masa poco calificado, pero el trabajo del cliente se parece más al artesanal que incluso trabaja con sus manos, lo que dificulta la estandarización del tiempo global de prestación del servicio (producción-circulación-consumo). Así mismo, el concepto de resistencia del trabajador debería ampliarse y no referirse solo a la gerencia sino al mismo cliente. Esta última forma de resistencia toma una forma eminentemente simbólica (Hall, 1997).

En cuanto a la flexibilidad: en el diseño de software, a causa de la dependencia del diseño de las habilidades del trabajador, se dificulta la flexibilidad numérica; en cambio en el McDonald's, el trabajo taylorizado en la parte propiamente de fabricación de la hamburguesa la facilita, no así en la venta. Por lo que respecta de la flexibilidad funcional, está se presenta como flexibilidad cognitiva en el software porque el diseñador puede participar simultáneamente en varios proyectos sin cambiar de puesto o departamento; en los McDonald's se facilita por la simplificación de las tareas y porque los empleados son capacitados para desarrollar cualquiera de estas; la presencia de bonos puede estar presente en ambos.

En síntesis la producción capitalista que implica trabajo no clásico históricamente ha encontrado obstáculos para su estandarización, por las dificultades de estandarizar la producción de símbolos, la propia interacción del empleado con 
el cliente y el trabajo del cliente. De tal forma que, paradójicamente, en el momento actual habría dos estrategias del capital en este tipo de trabajador, una la de estandarizar aquello que no había sido posible y otra arriesgarse a dar más poder de decisión al empleado que a su contrapartes en el trabajo físico material de la industria y agricultura.

\section{TRABAJO NO CLÁSICOY SUBCONTRATACIÓN}

Cuando se inició en la década del ochenta la discusión actual sobre la flexibilización del trabajo, la subcontratación aparecía como un anexo de la misma; el acento se ponía al interior del proceso de trabajo. Para los noventa la subcontratación pasó al primer plano de la flexibilidad, relacionada con el concepto de empresa red y también con el de cadena de valor, clúster y distrito industrial. La subcontratación se ha facilitado desde los noventa por la informatización (Taylor, 2005). En la actualidad se debate si la subcontratación es el paradigma productivo que nacerá de la crisis actual. Por supuesto que hay varias circunstancias de subcontratación: la de tareas complementarias a la principal actividad de transformación de la empresa, tanto en bienes como en servicios - la contabilidad, el diseño, la comercialización, el cosido de pantalones, etc. - o bien si se subcontratan tareas del core de las actividades que definen a una industria. Hay quienes plantean que solo conviene a la empresa lo primero y debería de dirigir directamente a los trabajadores en el segundo caso para garantizar calidad, productividad, lealtad, identidad con la empresa. Un problema complementario es el uso de agencias de contratación de personal, que no dirigen a los trabajadores productivamente sino que los seleccionan, los contratan como si fueran de la propia agencia y los destinan a trabajar en la compañía que subcontrata con esta. Las consecuencias generales de la subcontratación serían la precarización de los empleos y la pérdida de fuerza de los sindicatos. Los trabajadores subcontratados en general tendrían jornadas laborales y riesgos en el trabajo mayores y una pérdida de derechos e identidad con la empresa (García, Mertens y Wilde, 1998).

Por supuesto que el trabajo no clásico se puede subcontratar, sin embargo, como es menos controlable, al depender la forma de la interacción y la creación de símbolos compartidos en buena medida del trabajador en su relación con el cliente, la subcontratación podría implicar una menor satisfacción del cliente (menor calidad del servicio) lo que marcaría limites a esta forma de flexibilización o bien la necesidad de que la empresa que subcontrata destine personal de supervisión directamente en las instalaciones de la subcontratada. Es el caso de call centers subcontratados por grandes corporaciones en donde estas llegan a establecer oficinas cerca de las mesas de trabajo combinando su supervisión con las de la empresa de call centers, lo que puede originar conflictos y órdenes que se contraponen. Cuando sea posible estandarizar las interacciones o la comunicación con clientes resultará más factible la subcontratación que en trabajos más sofisticados de creación de confianza con el cliente o de códigos o configuraciones simbólicas más profundas. Es decir, la "fábrica de sonrisas" tiene también su límite en la percepción de la sinceridad que puede no lograr la confianza del consumidor en la oferta del producto.

\section{SUJETOS NO CLÁSICOS, IDENTIDAD Y FRAGMENTACIÓN ¿SERVIDUMBRE VOLUNTARIA?}

A mediados de los noventa surgió una teoría que, derivada de la del fin del trabajo de la década anterior y de la postmodernidad, planteó en términos sociológicos - supuestamente demostrable empíricamente - que la inestabilidad de las ocupaciones en la Nueva Economía se traducía en trayectorias laborales fragmentarias - ocupaciones desvinculadas y fugaces -, como nueva caracterís- 
tica del mercado laboral y que la decadencia del Estado benefactor junto con sus sistemas de relaciones industriales, implicaría una pérdida de identidad con el Trabajo (Dubar, 2001; Dubar 2002). En esta medida ya no serían concebible la conformación de sujetos laborales amplios, ni organizaciones ni proyectos de transformación de la sociedad (Geiser, 1997; Kirk y Wall, 2011).

Un problema de esta tesis es que en lo fundamental hace derivar la identidad colectiva y personal de la posición en la ocupación (Parsons, 1968), es decir, ante la heterogeneidad de las ocupaciones resulta una no identidad, y ante trayectorias ocupacionales zigzagueantes, no coherentes, tampoco habría identidad a nivel individual con algún tipo de trabajo en particular (Dreher et al., 2007; Dubar, 1991). Sin embargo, hacer depender la identidad de la ocupación remite a un pasado artesanal ya muy lejano. En las grandes empresas, desde la revolución industrial, la identidad no tenía que ver con cada ocupación en particular, mucho menos con la extensión del taylorismofordismo (Bizberg, 1989; Burke et al. 2009; Cerullo, 1997). Cuandollega el toyotismo y la automatización, por el contrario, la propia empresa busca inducir identidad colectiva con ella y el trabajador automatizado, computarizado e informatizado es posible que haya reeditado en términos modernos un orgullo de trabajar con tecnologías de punta (De la Garza, 2007; Dubet, 1989).

Es decir, la identidad colectiva de los trabajadores no terminó con el obrero de oficio, se siguió en casi todo el siglo XX con identificaciones no siempre con el producto o la actividad realizada sino con sus compañeros, sus organizaciones o sus partidos. Además, es probable que sobre la identidad de los trabajadores no solo influya la actividad laboral desarrollada, sino otros espacios de la vida no laboral como la familia, el ocio, la religión, el espacio urbano o rural (Beriani y Pataxi, 1996; Eagleton, 2006; Giddens, 1991). Por otro lado, la identidad debería de considerarse como una forma de la subjetividad (Gimenez, 2008, 1992, 1996; Hogg, Terry y White, 1995; Howard, 2000), en tanto proceso de crear el sentido de identificación, pero situado en ciertas estructuras y en determinadas interacciones con otros actores (Linhart, 2008; Melucci, 2001). Vista como subjetividad podría ser pensada como una configuración de códigos cognitivos, morales, emotivos, estéticos y formas de razonamiento cotidiano que permiten dar identidad por y para (Schutz, 1996, 2003, 2003a). Entre el puesto de trabajo y la identidad se encuentran otras estructuras (al interior de la empresa, organizacionales, tecnológicas, de relaciones laborales, de ocupaciones, de calificaciones, culturales; al exterior de cadenas entre clientes y proveedores, del mercado de trabajo, del mercado del producto, cadenas productivas). El trabajador en un puesto se encuentra en interacción con sus compañeros, supervisores y jefes. Finalmente, el proceso de creación de identidad pone en juego códigos como los mencionados provenientes de la cultura (Taylor, 1989; Portal, 1991), pero los sujetos obreros construyen las configuraciones específicas de sentido para situaciones concretas (Sainssaulieu, 1977). Estas pueden cambiar en función de estructuras como las mencionadas pero también de experiencias (Sewell, 1992). En otras palabras, sobre la formación de identidad influyen procesos más complejos que las simples características del puesto de trabajo (Taylor, 1992; De Gaulejac, 1993).

Por otro lado, los procesos colectivos de identificación no conducen a formas que deban permanecer; ya quela identidad puede crearse, ser contradictoria y parcial (Recour, 1992; Pizzorno, 1983; Mead, 1972) y a la vez desestructurarse; cuando esto sucede no significa que esta situación llegó para quedarse, como plantea Sennet. En el caso de los trabajadores asalariados del capital, además de las estructuras, subjetividades, interacciones en el trabajo y la influencia de otros espacios de la vida (Gayosso, 2011), no hay que olvidar que el eje es la acumulación del capital y, en esta medida, pueden entrar en contradicción dicha acumulación con las aspiraciones, con lo que consideran un trabajo legítimo los propios trabajadores y desencadenar un proceso que afiance la identidad e incluso la constituya desde 
formas muy ambiguas en torno del propio movimiento (Retamozo, 2006).

El trabajo en los servicios puede implicar relaciones individualizadas con el cliente, la generación de interacciones y de símbolos compartidos, depender en buena medida del trabajador y en este sentido dificultar la construcción de identidad colectiva. Esta sería otra forma de la conocida tesis de Bauman de fragmentación de identidades, que en última instancia dependería de las características del puesto de trabajo. Sin embargo, la "fábrica de sonrisas" iría desde la alta especialización en interacción con clientes y creación de símbolos cognitivos, morales, emotivos o estéticos compartidos, hasta el acercamiento a la estandarización de dichas interacciones y símbolos sin mayor profundidad en las relaciones (por ejemplo la oferta de un producto por teléfono puede hacerlo una grabación o cuando lo hace un trabajador usar frases ya hechas y tener respuestas estandarizadas, aunque, como mencionamos al entablarse la interacción, el cliente puede fácilmente salirse del guión que ante un trabajador poco hábil la interacción puede salirse de control).

Entre los trabajadores no clásicos de alta calificación, esta puede significar capacidades técnicas para resolver un problema, pero también calificación en cómo lograr una interacción y simbolización que lleven al cliente a aceptar el producto o la explicación que se le ofrece. Entre los de baja calificación - cajeros y acomodadores de Wal Mart, empleados de restaurante de McDonald's, trabajadores de call centers, cajeros de bancos), aunque de ellos depende finalmente la venta o generación del servicio, este puede estar de tal forma estandarizado que la actividad se vuelva rutinaria, estándar, simple y de baja remuneración ,y la construcción social de la identidad, puede basarse en el reconocimiento compartido de su situación de precariedad (Lomnitz, 1998; Ramírez, 2005); es decir, no resultar solo del puesto de trabajo sino de una en compleja configuración como ya hemos explicado. Tampoco resulta imposible la identificación de los expertos y técnicos en interacciones y creación de símbolos (por ejemplo los artistas exclusivos que muchas veces se adhieren a causas que consideran justas), aunque la alta especialidad si mueve hacia el individualismo en las soluciones laborales y de la vida, aunque tampoco hay determinismo en este sentido: en determinadas coyunturas el individualismo puede sumergirse frente a eventos impactantes socialmente como ha sucedido durante las revoluciones.

En síntesis, la importancia actual de los trabajos no clásicos tendría que llevarnos a revisar el concepto de clase social, de conflicto de clases, de sujetos trabajadores, de organizaciones y de proyectos (Dubet, 1999), en lugar de suponer en forma superficial que entre estos ya no son posibles las solidaridades, acciones y organizaciones colectivas (De Jours, 1998; Linhart, 2009) y que el futuro queda determinado por la sumisión voluntaria (aceptación del control empresarial del trabajo por voluntad del proprio trabajador, sin coersión).

Recebido para publicação em 18 de janeiro de 2013 Aceito para publicação em 27 de maio de 2013

\section{REFERENCIAS}

BERIANI, Josetxto; A. Patxi. Identidades culturales. Bilbao: Universidad de Deusto. 1996. 220p.

BIZBERG, Ilán. Individuo, identidad y sujeto. Estudios Sociológicos. México, v. 7, n. 21, sept./dic., p. 485-518 1989.

BURKE, Peter, et al. (Ed.). Advances in identity theory and research. N.Y.: Klewer Academic 2009.

BURAWOY, Michael. Manufacturing consent. Chicago: University of Chicago Press, 1979. 253p.

CERULLO, J. Identity Construction: new issues, new directions. Annual Review of Sociology, 23, p. 385-409, 1997.

DE GAULEJAC, Vincent. El costo de la excelencia. BuenosAires: Paidós, 1993. 285p.

DE JOURS, C. Souffrance en France: banalisation de l'injustice sociale. Paris: Seuil, 1998. 225p.

DE LA GARZA, Enrique. Trabajo y mundos de vida. In: ZEMELMAN, H. (coord.) Subjetividad: umbrales del pensamiento social. Barcelona: Anthropos, 1997.

La epistemología crítica y el concepto de configuración. Revista Mexicana de Sociología. México, D.F., v. 63, n. 1, enero-marzo, p.109-127, 2001. 
¿Hacia dónde va la Teoría Social? In: Tratado Latinoamericano de Sociología Latinoamericana. Barcelona: Anthropos; México: Universidad Autónoma Metropolitana. 2006. 318p.

Hacia un concepto ampliado de trabajo. Barcelona: Anthropos, 2010. 157p.

La querella de las identidades. In: Trabajo no clásico, identidad y acción colectiva. México, D.F.: Plaza y Valdés-CLACSO, 2011.

et al. Crítica de la razón parapostmoderna. $R e$ vista Latinoamericana de Estudios del Trabajo, v. 13, n. 19, 2007.

DREHER, J. et al. (comps.) Construcción de identidades en sociedades pluralistas. B.A.: Lumiere, 2007.

DUBAR, C. La socialisation. Construction des identités sociales et professionalles. París: Armand Collin Ed, 1991.

El trabajo y las identidades profesionales personales. Revista Latinoamericana de Estudios del Trabajo, v.7, n. 13, 2001.

La crisis de las identidades. Barcelona: Ed. Bellatorre, 2002

DUBET, François. ¿En qué sociedad vivimos? Buenos Aires: Lozada, 1999.

De la sociología de la identidad a la sociología de sujeto. Estudios Sociológicos, v. 7, n. 21, sept./dic., 1989 DURAND, J. P. La chaine invisible. París: Seuil, 2004

. La question du consentement au travail. Paris L'Hartmahan, 2006

EAGLETON, T. La estética como ideología. Madrid: Trotta Editores, 2006.

GARCÍA, A., L. Mertens y L. Wilde. Procesos de subcontratación y cambios en las calificaciones de los trabajadores. Santiago: CEPAL, 1998.

GARCÍA, B. La situación laboral actual: marcos conceptuales y ejes analíticos pertinentes. Trabajo, n. 3, dic. 2006.

GAYOSSO, Jose Luis. Trabajo, identidad y acción colectiva en los comerciantes artesanos del Centro Histórico de Coyoacán. En: DE LA GARZA, E. (Coord.). Trabajo no ๓ clásico. Organización y Acción Colectiva. México, D.F. จ Plaza y Valdés-UAM, 2011.

$\therefore$ GEISER, Marcela. Identidad, subjetividad y sentido común en sociedades complejas. México, D.F.: Juan Pablos, 1997 GIDDENS, Anthony. Modernidad e identidad del Yo. Barcelona: Península, 1991.

GIMÉNEZ Montiel, G. Materiales para una teoría de las identidades sociales.[Versión electrónica]. Frontera Norte, v. 9 , n.18, p. 1-14, 1997. http:/wwwlaramabiblioteca.com. transforcurr/GIMENEZ\%20Materiales\%20para\%2 . transforcurr/GIMENEZ\%20Materiales\%20para\%2 sociales.pdf. Acesso em 2 de marzo de 2008.

Gilberto Estudios sobre la cultura y las identidades sociales. México, D.F.: Conaculta-Iteso, 2008.

La identidad social o el retorno del sujeto en sociología. En: MERCADO, L. (coord.). Identidad, análisis, teoría, simbolismo. México, D.F.: UNAM, 1996.

GIMENEZ, Gilberto et al. Teoría y análisis de la identidad social. Reseñas Bibliográficas. México, D.F.: UNAM, 1992.

GODOLIER, Maurice. Lo ideal y lo material. Madrid: Taurus Humanidades, 1989

HALL, Stuart. Who Needs Identity? In: HALL, S y GAY, Paul. Questions of cultural identity. London: Saje, 1997.
HANDY, C. El futuro del trabajo humano. Buenos Aires: Ariel, 1986.

HOCHSCHILD, A. R. The managed heart: Commercialization of Human Feeling. N.Y. Hammondsworth-Penguin, 1983.

HOGG, M. A., TERRY; D.; WHITE, K. A tale of two theories: a critical comparation of identity theory with social identity theory. Social Psychology Quarterly, v. 58, n.4, p. 255-69, 1995.

HOWARD, J. Social Psychology of Identities. Annual Review of Sociology, n. 26, p. 367-93, 2000.

JURGENS, U. Shaping the Future of Work. British Journal of Industrial Relations, v. 33, n.4, dic., 1995.

KIRK, J.; WALL, C. Work and Identity. London: MacMillan, 2011.

KOREZYNSKI, M; HODSON, R.; EDWARDS, P. Socia theory at work. Oxford: Oxford University Press, 2006.

LINHART, D. Porque le travillons-nous, une approche sociologique de la subjectivité aun travail. Paris: ERES, 2008.

Travaller sans les Autres. Paris: Sauil, 2009.

LOMNITZ, L. Cómo sobreviven los marginados (14va ed.) México: Siglo XXI, 1998.

LURIA, Alexander; LEONTIEV, Alexis; VIGOTSKY, Lev Semenovich. Psicología y pedagogía. Madrid: Akal, 2004.

MARTÍNEZ, Aleida. Subcontratación y Calidad de los Empleos. Tesis en Opción al Grado de Maestra en Gobierno. México, D.F.: FLACSO, 2008.

MEAD, George. Mind, Self and Society. Chicago: University of Chicago Press, 1972.

MELUCCI, A. Challenging Codes. Collective action in the information age. Cambridge: Cambridge University Press, 2001.

MICHELI, J. El Trabajo en la Sociedad de la Información. Estudios Sociológicos, v. 24, n. 70, enero-abril, 2006.

MONCADA, M. MONSALVO, J. Implicaciones laborales del outsourcing. Tesis en Opción al Grado de Abogados. Bogotá: Pontificia Universidad Javeriana, 2000.

MOORE, H.L. The Future of Work. British Journal of Industrial Relations, v. 33, n.4, dic. 1995.

MUCKENBERGER, U. Towards a new definition of employment relationship. International Labor Review, v. 135, n. 6, 1996

NOVELLA, B. et al. Off shoring and the labour market. Ginebra: ILO, 2007.

OFFE, C. La sociedad del trabajo, problemas estructurales y perspectivas de futuro. Madrid: Alianza, 1998.

PARSONS, T. The position of identity in the general theory of action. In: GORDON, C; GEORGEN, K. (eds.). The self in social interactions. N.Y.: Wiley, 1968.

PIORE, M.; SABEL, P. La segunda ruptura industrial. Barcelona: Alianza Editorial, 1988.

PIZZORNO, A. Identitá e interesse. In: SCIOLLA, Loredana (ed.). Identitá. Turín: Rosenberg y Sellier, 1983.

POGLIAGHI, Leticia. La problemática del trabajo, la identidad y la acción colectiva en las taxistas de la ciudad de México. In: DE LA GARZA, E. (coord.), Trabajo no clásico, organización y acción colectiva. México, D.F.: Plaza y Valdés-UAM, Tomo II, 2011.

PORTAL, Mario. La identidad como objeto de estudio de la antropología. Alteridades, v. 1, n. 2, 1991. 
PORTES, A. En torno de la informalidad. México, D.F.: Miguel A. Porrúa, 1995.

RAMÍREZ, Kuri. Ciudadanía y participación en el espacio local de la ciudad de México. Ponencia presentada en el IV Congreso de IGLOM, México, D.F., 12 de nov. 2005.

RECOUR, P. Oneself as another. Chicago: The Chicago University Press, 1992.

REGLIA, Ido; Lavori Atipici, Nuovi, Non Standard. Sociología del Lavoro, 97, p. 38, 2003. RETAMOZO, M. El Movimiento de los desocupados en Argentina. Tesis de doctorado en investigación en ciencias sociales, FLACSO, 2006

SAINSSAULIEU, Renaud. L'identité au travail. Paris: References, 1977.

SALAS, C. El Sector informal. In: De la Garza, E. (coord.). Teorías sociales y estudios del trabajo. Barcelona: Anthropos, 2006.

SENISE, M. E. Empleo atípico y mercado de trabajo atípico. Granada: Facultad de Ciencias Económicas y Empresariales, Universidad de Granada, 2001.

SENNET, Richard; Coob, J. The Hidden injures of class. N.Y.: Knopt, 1972.

SEWELL, William. A theory of structure: duality, agency and transformation. The American Journal of Sociology, v. 98, n. 1,p. 1-29 jul., 1992.
SHUTZ, A. Fenomenología del mundo social. Buenos Aires: Paidós, 1996.

SHUTZ, A. El problema de la realidad social. Buenos Aires: Amorrortu, 2003.

Estudios sobre la teoría social. Buenos Aires: Āmorrortu, 2003a.

TAYLOR, Charle. Sources of the self: the making of modern identity. Harvard: Harvard University Press, 1989.

TAYLOR, T. In defense of outsourcing. Cato Journal, Spring, n. 2, 2005.

TAYLOR, V. Collective identity in social movement communities. In: MORRIS, A. D. (comp.). Frontiers in social movement theory. Yale: Yale University Press, 1992.

THOMPSON, Edward. La formación de la clase obrera en Inglaterra. Barcelona: Laia, 1972.

THOMPSON, P. The nature of work. Londres: McMillan, 1983.

TOKMAN, V. El sector informal hoy. Chile: OIT, 1987.

URIARTE, U.; TUSSO, N. Descentralización, tercerización y subcontratación. Montevideo: OIT, 2009.

ZUCCHETTI, E. Un mercato del lavoro plurale. Sociología del Lavoro, n. 97, p. 25, 2003. 


\section{NON-CLASSICAL LABOR AND FLEXIBILITY}

\author{
Enrique de la Garza Toledo
}

In this article we will present the concept of non-classical labor (De la Garza, 2010) in an attempt to explain not only the differences among types of work, but also to address broader dimensions, which are partially included in other theories (Thompson, 1983). We will attempt to clarify the concept and its scope, as well as the relations with the services activities, which have gained constantly increasing importance in modern economies, even in less developed ones. We will recall the classical concept of flexibility (Durand 2004) and the extent to which it would be pertinent to include non-classical labor. More specifically, we will include a form of flexibilization which has probably become dominant in capitalism since the most recent great economic crisis, outsourcing (Moncada e Monsalvo, 2000) and its connections with non-classical labor. Lastly, we will discuss the thesis of fragmented identities (Sennett e Cobb, 1972) and voluntary servitude (Durand, 2006) in the vein of the approach mentioned in the nonclassical article, culminating in considerations on the possibility of training subjects who work under these conditions.

Key WoRDs: Work. Classical labor. Non-classical labor.

\section{TRAVAIL NON CLASSIQUE ET FLEXIBILITÉ}

\author{
Enrique de la Garza Toledo
}

Nous présentons dans cet article le concept de travail non-classique (De la Garza, 2010), pour essayer d'expliquer non seulement les différences entre les types de travail mais aussi d'étudier les larges dimensions inclues, en partie, dans d'autres théories (Thompson, 1983). Nous essayons d'expliquer le concept et ses portées ainsi que les relations avec les activités de service, qui sont devenues de plus en plus importantes dans les économies modernes, sans oublier son poids dans celles moins développées. Nous rappelons le concept classique de flexibilité (Durand, 2004) et l'extension qu'il serait bon d'inclure dans le travail non-classique. Nous introduirons tout particulièrement une forme de flexibilité qui va probablement devenir dominante dans le capitalisme, suite à la dernière grande crise économique, les prestations de services (Moncada et Monsalvo, 2000) et leurs liens avec le travail non-classique. Au final, nous discuterons de la thèse des identités fragmentées (Sennett et Cobb, 1972) et de la servitude volontaire (Durand, 2006) en fonction de l'approche mentionnée du travail non-classique pour en arriver à des considérations sur la possibilité de formation des personnes qui travaillent dans ces conditions.

Mots-CLÉs: Travail. Travail classique. Travail non classique.

Enrique de la Garza Toledo - Doutor em Sociologia. Professor da Universidade Autônoma Metropolitana do México, Departamento de Sociologia. Professor visitante da Universidade Autônoma de Barcelona, Universidade de Cornell e de Evry (França). Pesquisador da Posgraduação em Estudios Laborales de la Universidad Autónoma Metropolitana en México. Publicações recentes: La revitalización del debate del proceso de trabajo. Revista Latinoamericana de estudios del trabajo. v. 16, n. 26, 2012; El trabajo no clásico y la ampliación de los conceptos de la Sociología del Trabajo. Revista de Trabajo. Dinámica del trabajo en el marco de la incertidumbre global, v 8, n. 10, Nueva Época, Buenos Aires, Argentina.2012 